\title{
Intracerebral hematoma from aneurysm rupture
}

\author{
Khalid M. Abbed, M.D., And Christopher S. Ogilvy, M.D. \\ Department of Neurosurgery, Cerebrovascular Surgery, Massachusetts General Hospital/Harvard \\ Medical School, Boston, Massachusetts
}

\begin{abstract}
Object. Patients who present with an intraparenchymal hematoma associated with a ruptured aneurysm usually require urgent clot evacuation and aneurysm obliteration. The impact of the presence of hematoma on outcome has been poorly characterized. The authors report on 460 patients who had dense subarachnoid hemorrhage (SAH) (Fisher Grades 3 and 4) with and without associated hematoma.

Methods. Of the 959 consecutive patients who presented with SAH, 460 patients with Fisher Grade 3 and 4 SAH were analyzed and divided into two groups: those with (Group 1) and those without (Group 2) hematoma. The presenting Hunt and Hess grade and 6-month outcomes of the two groups were compared.

Of the 460 patients, $116(25 \%)$ had intraparenchymal hematomas and admission Hunt and Hess grades were worse in Group 1 compared with Group 2. Outcome scores were worse for Group 1 compared with Group 2; however, when comparing Group 1 and Group 2 within the same initial Hunt and Hess score, there was no statistical difference in outcome.

Conclusions. Intraparenchymal hematoma in association with SAH does not differ significantly from those patients without associated hematomas. We therefore recommend aggressive clot evacuation and aneurysm obliteration.
\end{abstract}

KEY WORDS - intracerebral hematoma - aneurysm rupture • subarachnoid hemorrhage

Although rupture of cerebral aneurysms usually results in SAH, some degree of intraparenchymal bleeding is often seen. A cooperative study reported by Locksley ${ }^{10}$ showed that $90 \%$ of patients with SAH who died within 72 hours also had intracerebral clots. Only 4 to $17 \%$ patients with aneurysmal SAH, however, have clinically significant hematomas. ${ }^{16}$

It is well known that the presence of an ICH after aneurysm rupture negatively influences the patient's clinical course and outcome. ${ }^{4,6}$ The overall mortality rate ranges from 36 to $58 \%$. $1,14,19$

Controversy persists regarding the optimal management of ICHs caused by aneurysm rupture..$^{2,11-13,17,19}$ In this paper, we review the literature and present our experience at the Massachusetts General Hospital with intraparenchymal and sylvian hematomas caused by ruptured aneurysms.

Abbreviations used in this paper: $\mathrm{ACA}=$ anterior cerebral artery; $\mathrm{ACoA}=$ anterior communicating artery; $\mathrm{CT}=$ computerized tomography; GOS = Glasgow Outcome Scale; ICA = internal carotid artery; $\mathrm{ICH}=$ intracerebral hematoma; $\mathrm{MCA}=$ middle cerebral artery; $\mathrm{PCoA}=$ posterior communicating artery; $\mathrm{SAH}=$ subarachnoid hemorrhage; VBA = vertebrobasilar artery.

\section{ANALYSIS OF THE MASSACHUSETTS GENERAL HOSPITAL EXPERIENCE}

Between 1991 and 2000, 959 patients were treated with aneurysmal SAH at the Department of Neurosurgery of the Massachusetts General Hospital. Of these 959 patients, 460 experienced dense bleeding (Fisher Grades 3 and 4), excluding subdural hematoma and intraventricular hemorrhage. Of these 460 patients, 314 were women and 146 were men, and their ages ranged from 21 to 86 years (Table 1).

TABLE 1

Age and sex distribution of patients with and without ICH

\begin{tabular}{crrrr}
\hline \hline & \multicolumn{2}{c}{ Group 1 } & & \multicolumn{2}{c}{ Group 2 } \\
\cline { 2 - 3 } \cline { 4 - 5 } Age (yrs) & $M$ & F & M & F \\
\hline $0-39$ & 5 & 5 & 18 & 36 \\
$40-49$ & 12 & 19 & 29 & 39 \\
$50-59$ & 6 & 25 & 29 & 61 \\
$60-69$ & 5 & 19 & 24 & 45 \\
$70-79$ & 3 & 10 & 12 & 37 \\
$\geq 80$ & 0 & 5 & 3 & 13 \\
total & 31 & 83 & 115 & 231 \\
\hline
\end{tabular}


TABLE 2

Site of ruptured aneurysm stratified by group

\begin{tabular}{lrc}
\hline \hline \multicolumn{1}{c}{ Site } & Group 1 & Group 2 \\
\hline MCA & 44 & 35 \\
ACoA & 41 & 110 \\
PCoA & 11 & 63 \\
distal ACA & 5 & 5 \\
ICA & 12 & 37 \\
VBA & 1 & 96 \\
total & 114 & 346 \\
\hline
\end{tabular}

The patients were divided into two groups based on an initial head CT scan. Group 1 comprised patients with a hematoma, and Group 2 comprised patients without a hematoma. On admission, each patient's neurological condition was graded according to the Hunt and Hess scale. ${ }^{7}$ All patients underwent both preoperative CT scanning and conventional angiography. All patients were treated surgically according to an aggressive treatment protocol of urgent clot evacuation and aneurysm clipping with or without hemicraniectomy. Six months after the SAH the outcome was assessed according to the GOS. ${ }^{9}$

\section{SITE OF RUPTURED ANEURYSM}

The most frequent site of aneurysm rupture in patients with an associated hematoma was the MCA (38\% of patients) followed closely by the ACoA (36\% of patients). The incidence of a hematoma was higher in patients with MCA (56\%) and distal ACA aneurysms (50\%), and it was lower in patients with ICA (24\%) and PCoA aneurysms $(15 \%)$. Intracerebral hematomas were very rare in patients with VBA aneurysms (1\%) (Table 2).

\section{RELATIONSHIP BETWEEN ANEURYSM SITE AND HEMATOMA LOCATION}

The types of ICH were classified into seven groups according to CT findings on admission: 1) frontal; 2) temporal, 3) sylvian; 4) basal ganglia; 5) interhemispheric; 6) callosal; and 7) cerebellar (Table 3).

The location of the ICH is related to the location of the ruptured aneurysm. In patients with MCA aneurysms, the sylvian fissure was by far the most common location for hematoma occurrence. Frontal and temporal lobe he-

TABLE 3

Aneurysm site stratified by hematoma location

\begin{tabular}{lrrrrrr}
\hline \hline \multicolumn{1}{c}{ Location } & MCA & ACoA & PCoA & $\begin{array}{c}\text { Distal } \\
\text { ACA }\end{array}$ & ICA & VBA \\
\hline frontal & 6 & 35 & 0 & 3 & 0 & 0 \\
temporal & 8 & 0 & 10 & 0 & 7 & 0 \\
sylvian & 29 & 0 & 1 & 0 & 1 & 0 \\
basal ganglia & 0 & 0 & 0 & 0 & 4 & 0 \\
interhemispheric & 0 & 7 & 0 & 0 & 0 & 0 \\
callosal & 0 & 0 & 0 & 2 & 0 & 0 \\
cerebellar & 0 & 0 & 0 & 0 & 0 & 1 \\
total & 43 & 42 & 11 & 5 & 12 & 1 \\
\hline
\end{tabular}

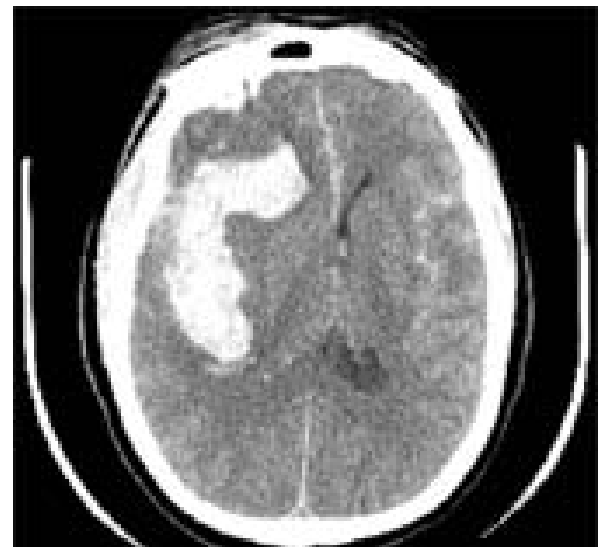

Fig. 1. A CT scan revealing a right frontotemporal hematoma secondary to a right MCA aneurysm rupture.

matomas also occurred in patients with MCA aneurysms (Fig. 1). In patients with ACoA aneurysms, frontal lobe hematomas occurred most frequently along with several cases of interhemispheric clots (Fig. 2). Temporal lobe hematomas were most frequent in patients with PCoA aneurysms (Fig 3). In patients with distal ACA aneurysms, ICHs were most commonly frontal and callosal in a typical butterfly-type pattern (Fig. 4). In patients with ICA aneurysms hematomas were most often found in the temporal lobe but were also associated with basal ganglia hematomas (Fig. 5) and one sylvian clot. Only one patient experienced a hematoma (fourth ventricle/cerebellar) that occurred in conjunction with a VBA aneurysm.

\section{NEUROLOGICAL CONDITION ON ADMISSION}

Hunt and Hess grades on admission were significantly higher in the patient group with ICH. In the ICH group, $64 \%$ of patients had Hunt and Hess Grade IV or

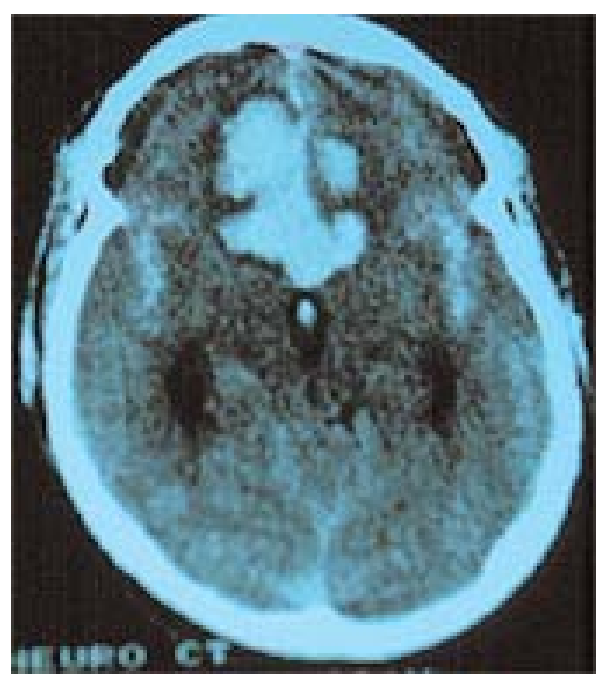

Fig. 2. A CT scan revealing interhemispheric and bilateral (right-left) inferior frontal lobe hematomas secondary to an ACoA aneurysm rupture. 


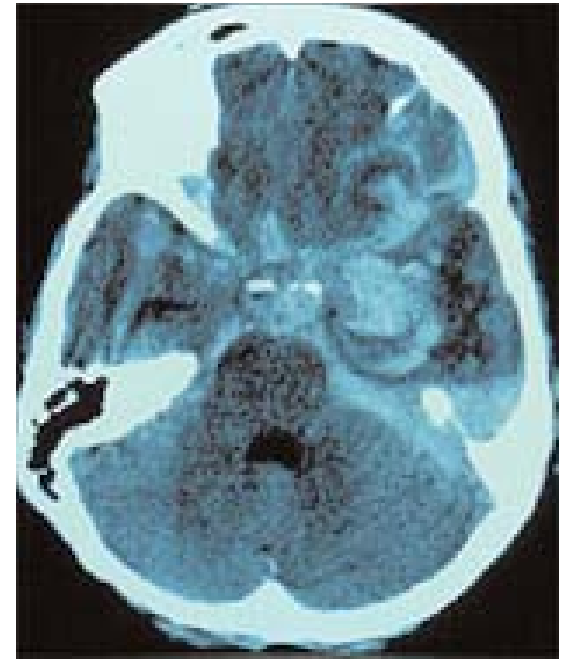

Fig. 3. A CT scan revealing a left temporal lobe hematoma secondary to rupture of a left PCoA aneurysm rupture.

$\mathrm{V}$ compared with $30 \%$ in the patient group without ICH (Table 4).

\section{OUTCOME 6 MONTHS AFTER SAH}

Similarly, GOS scores were significantly worse in patients with ICH when compared with patients without ICH. In the ICH group, 37\% of patients had an excellent or good outcome compared with $61 \%$ in the group without ICH. Also, $45 \%$ of the patients with ICH had a poor outcome or died compared with $27 \%$ of the patients without ICH (Table 5).

When comparing patients with SAH with and without ICH who had the same Hunt and Hess grade, there was no significant difference in outcome (Figs. 6 and 7).

\section{DISCUSSION}

In this retrospective analysis, the incidence of ICH demonstrated on initial head CT scanning was $12 \%$,

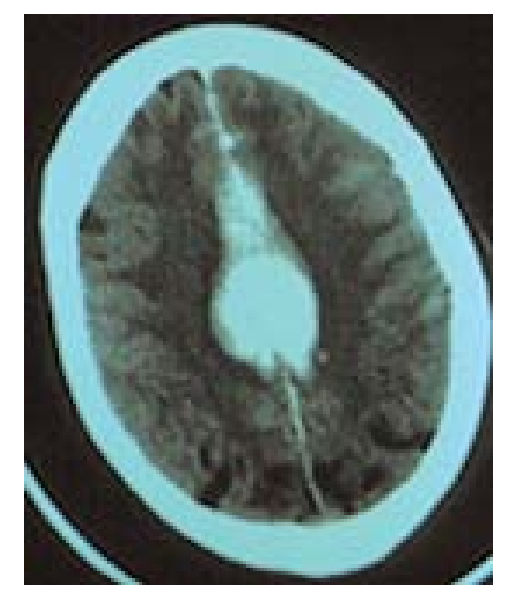

Fig. 4. A CT scan revealing a butterfly-pattern interhemispheric hematoma secondary to rupture of a distal ACA aneurysm.

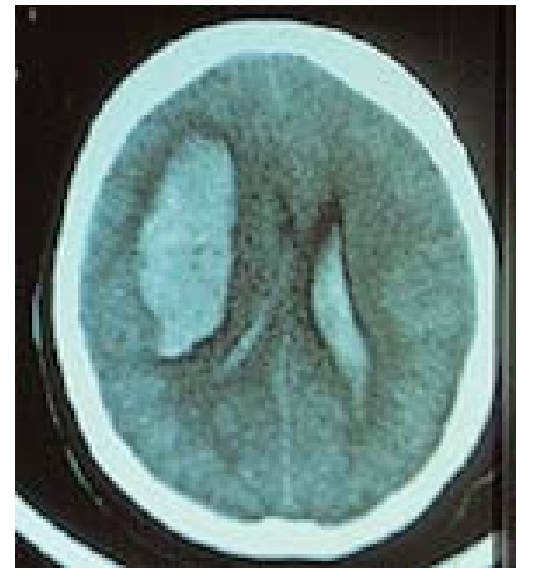

Fig. 5. A CT scan revealing a temporal lobe/basal ganglia hematoma (with ventricular extension) secondary to an ICA aneurysm rupture.

which is consistent with the figures in previously published studies (4-19\%)..$^{5,13,15,18}$ The fact that the most frequent sites of aneurysm rupture in patients with $\mathrm{ICH}$ were the MCA and ACoA and that the highest incidence of $\mathrm{ICH}$ was in patients with MCA and distal ACA aneurysms was also consistent with previously published data. ${ }^{8,14,17,18}$

Patients with SAH and an associated intraparenchymal or sylvian hematoma present with worse Hunt and Hess grades. In our series, $2 \%$ of patients with ICH presented as Grade I, $3 \%$ as Grade II, $31 \%$ as Grade III, $49 \%$ as Grade IV, and $15 \%$ as Grade V. In contrast, $15 \%$ of the group without an associated hematoma presented as Grade I, 7\% as Grade II, $48 \%$ as Grade III, $24 \%$ as Grade IV, and $6 \%$ as Grade V (Table 4).

Similarly, it has been shown that patients with an ICH from ruptured aneurysms have worse outcomes. ${ }^{4,6}$ In our series, the GOS scores at 6 months posthemorrhage were as follows: $22 \%$ of patients with ICH had an excellent outcome, $15 \%$ had a good outcome, $17 \%$ had a fair outcome, $11 \%$ had a poor outcome, and $34 \%$ died. This mortality rate is slightly lower than previously published figures (36-58\%). ${ }^{1,14,19}$ In contrast, $40 \%$ of the group without an associated ICH had an excellent outcome, $21 \%$ had a good outcome, $12 \%$ had a fair outcome, $5 \%$ had a poor outcome, and 22\% died (Table 5).

When comparing the patients with SAH with and without ICH who had the same Hunt and Hess grade, there was no significant difference in outcome (Figs. 6 and 7). This shows that the poor outcome in patients with an ICH

TABLE 4

Admission Hunt and Hess grades by patient group

\begin{tabular}{rrr}
\hline \hline Grade & Group 1 & Group 2 \\
\hline I & 2 & 52 \\
II & 3 & 25 \\
III & 36 & 167 \\
IV & 56 & 82 \\
V & 17 & 20 \\
total & 114 & 346 \\
\hline
\end{tabular}


K. M. Abbed and C. S. Ogilvy

TABLE 5

Patient groups and GOS scores

\begin{tabular}{ccc}
\hline \hline GOS & Group 1 & Group 2 \\
\hline 1 & 34 & 66 \\
2 & 11 & 15 \\
3 & 17 & 36 \\
4 & 15 & 64 \\
5 & 22 & 119 \\
total & 99 & 300 \\
\hline
\end{tabular}

from aneurysm rupture may be related to their poor clinical state on admission. This finding is consistent with the findings of Tokuda, et al., ${ }^{18}$ but contrary to the results of Brott and Mandybur ${ }^{3}$ who found that the degree of bleeding predicted the outcome more accurately than clinical grade.

Controversy persists regarding the optimal management of ICH caused by aneurysm rupture. Tindall and Odom $^{17}$ recommended immediate emergency surgery with hematoma evacuation and aneurysm obliteration for patients with ICH secondary to aneurysm rupture. Lougheed and Marshall ${ }^{11}$ recommended emergency surgery for hematoma evacuation via burr hole in patients with elevated intracranial pressure. Papo, et al., ${ }^{13}$ have stated that some patients with Hunt and Hess Grade 5 may improve without intervention or after placement of an external ventricular drain and are then taken to the operating room for delayed surgery. Wheelock, et al., ${ }^{19}$ retrospectively reviewed data from 11 hospitals and obtained data on 132 patients with ICH caused by aneurysm rupture. They reported that patients treated with evacuation of the $\mathrm{ICH}$ without clipping of the aneurysm in the same operation had a mortality rate of $75 \%$. This mortality rate was de-

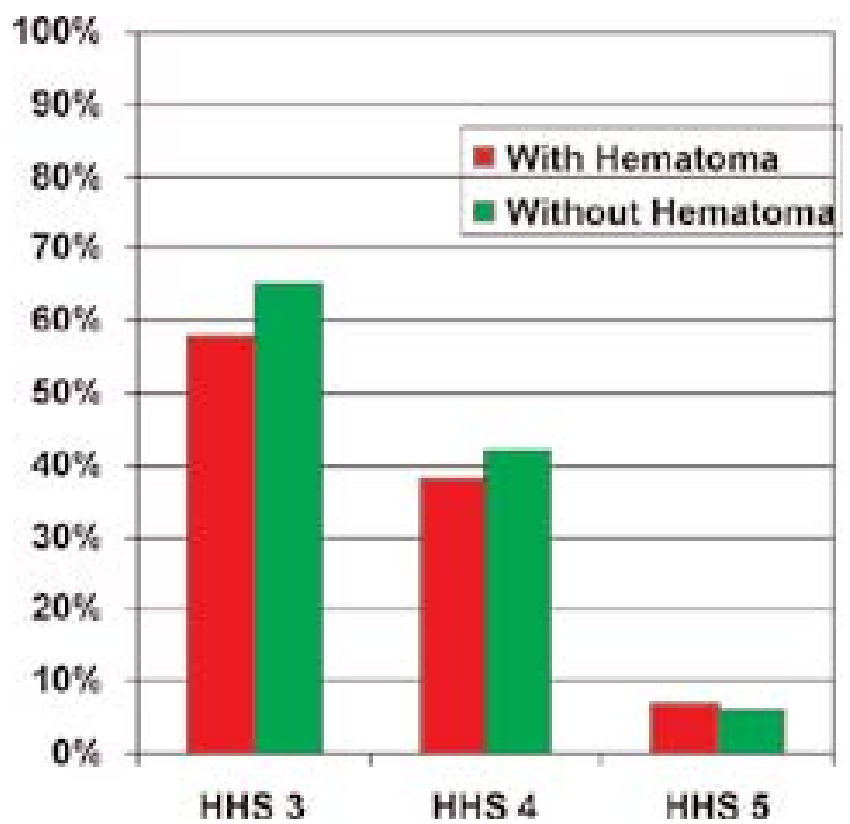

Fig. 6. Graph showing the percentage of good and excellent outcomes in higher Hunt and Hess grade SAHs.

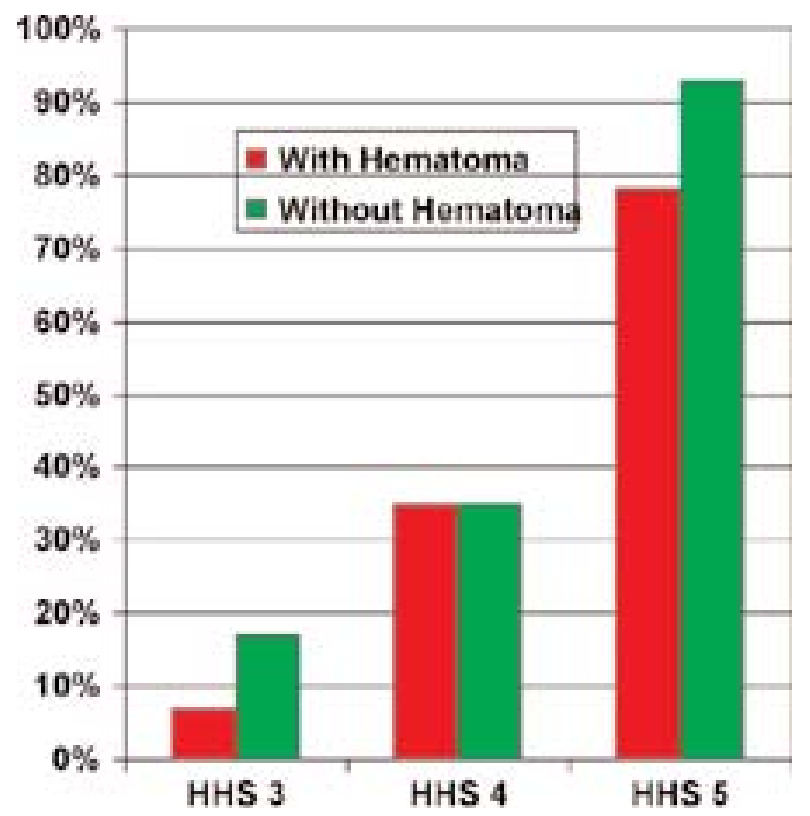
SAH.

Fig. 7. Graph showing mortality rates stratified by higher grade

creased to $29 \%$ if the aneurysm was obliterated during the same operation.

Based on the available evidence, patients with aneurysmal SAH, a large hematoma, and poor clinical grade have a poor prognosis regardless of therapy; however, we believe that early decompressive craniotomy with hematoma evacuation with simultaneous aneurysm clipping can salvage some critically ill patients.

\section{References}

1. Bohm E, Hugosson R: Experiences of surgical treatment of 400 consecutive ruptured cerebral arterial aneurysms. Acta Neurochir 40:33-43, 1978

2. Brandt L, Sonesson B, Ljunggren B, et al: Ruptured middle cerebral artery aneurysm with intracerebral hemorrhage in younger patients appearing moribund: emergency operation? Neurosurgery 20:925-929, 1987

3. Brott T, Mandybur TI: Case-control study of clinical outcome after aneurysmal subarachnoid hemorrhage. Neurosurgery 19: 891-895, 1986

4. Crompton MR: Intracerebral haematoma complicating ruptured cerebral berry aneurysm. J Neurol Neurosurg Psychiatry 25: 378-386, 1962

5. Gruss P: Therapeutic procedures in aneurysms with intracerebral hematoma, in Schiefer W, Klinger M, Brock M (eds): Advances in Neurosurgery. Berlin: Springer-Verlag, 1981, Vol 9, pp 271-280

6. Hijdra A, van Gijn J: Early death from rupture of an intracranial aneurysm. J Neurosurg 57:765-768, 1982

7. Hunt WE, Hess RM: Surgical risk as related to time of intervention in the repair of intracranial aneurysms. J Neurosurg 28: 14-20, 1968

8. Inagawa T, Hirano A: Ruptured intracranial aneurysms: an autopsy study of 133 patients. Surg Neurol 33:117-123, 1990

9. Jennett B, Bond M: Assessment of outcome after severe brain damage. A practical scale. Lancet 1:480-484, 1975

10. Locksley HB: Natural history of subarachnoid hemorrhage, intracranial aneurysms and arteriovenous malformations. Based 
on 6368 cases in the cooperative study. J Neurosurg 25:219 239, 1966

11. Lougheed WM, Marshall BM: Management of aneurysms of the anterior circulation by intracranial procedures, in Youmans JR (ed): Neurological Surgery: A Comprehensive Reference Guide to the Diagnosis and Management of Neurosurgical Problems. Philadelphia: WB Saunders, 1973, Vol 2, pp 731-767

12. Mohr G, Ferguson G, Khan M, et al: Intraventricular hemorrhage from ruptured aneurysm. Retrospective analysis of 91 cases. J Neurosurg 58:482-487, 1983

13. Papo I, Bodosi M, Doczi T: Intracerebral haematomas from aneurysm rupture: their clinical significance. Acta Neurochir 89: 100-105, 1987

14. Pasqualin A, Bazzan A, Cavazzani P, et al: Intracranial hematomas following aneurysmal rupture: experience with 309 cases. Surg Neurol 25:6-17, 1986

15. Sano K: Intracerebral haematomas, in Pia HW, Langmaid C, Zierski J (eds): Cerebral Aneurysms. Advances in Diagnosis and Therapy. New York: Springer-Verlag, 1979, 402-407

16. Tapaninaho A, Hernesniemi J, Vapalahti M: Emergency treat- ment of cerebral aneurysms with large haematomas. Acta Neurochir 91:21-24, 1988

17. Tindall GT, Odom GL: Saccular aneurysms of the brain. Surgical treatment, in Vinken PJ, Bruyn GW (eds): Handbook of Clinical Neurology. Vascular Diseases of the Nervous System, Part II. Amsterdam: North-Holland, 1972, Vol 12, pp 205-226

18. Tokuda Y, Inagawa T, Katoh Y, et al: Intracerebral hematoma in patients with ruptured cerebral aneurysms. Surg Neurol 43: 272-277, 1995

19. Wheelock B, Weir B, Watts R, et al: Timing of surgery for intracerebral hematomas due to aneurysm rupture. J Neurosurg 58:476-481, 1983

Manuscript received September 15, 2003.

Accepted in final form October 1, 2003.

Address reprint requests to: Christopher S. Ogilvy, M.D., Department of Neurosurgery, Massachusetts General Hospital, 55 Fruit Street, Vincent-Burnham Building Suite 710, Boston, Massachusetts 02114. email: cogilvy@ partners.org. 